\title{
Place des études post-AMM dans le suivi des risques du médicament : cahier des charges et méthodologies
}

\author{
Florence Tubach ${ }^{1}$, Véronique Lamarque-Garnier ${ }^{2}$, Anne Castot ${ }^{3}$ et les participants à la Table Ronde $N^{\circ} 5$ \\ de Giens XXVI
}

1 AP-HP, Centre de Pharmaco-épidémiologie, Paris, France; AP-HP, Hôpital Bichat, Département d'Épidémiologie Biostatistique et Recherche Clinique, Paris, France ; Université Paris Diderot, Sorbonne Paris Cité, France ; INSERM, CIE 801, Paris, France

2 Direction Évaluation de Santé, Sécurité et Gestion du Risque, Pfizer, Paris, France

3 Département de la Surveillance du Risque, du Bon Usage et de l'Information sur les Médicaments, Afssaps, Saint Denis, France

Texte reçu le 8 avril 2011 ; accepté le 30 mai 2011

\begin{abstract}
Mots clés :
études

de sécurité

post-AMM ;

risque

médicamenteux ;

plans de gestion

de risque ;

pharmaco-épidémiologie

Résumé - Les études réalisées après l'autorisation de mise sur le marché (AMM) ayant pour objectif d'identifier, caractériser ou quantifier un ou des risques, appelées Post-authorization safety studies (PASS), ont été renforcées ces dernières années, avec la mise en place des plans de gestion de risque (PGR), dont le cadre réglementaire européen a été défini en 2005 et qui vient d'être modifié récemment dans le cadre de la révision communautaire. Ces études de sécurité, interventionnelles ou non, portent sur un médicament commercialisé, qu'il soit ou non utilisé dans les conditions de l'autorisation de mise sur le marché (AMM). En dehors de ces études de sécurité, d'autres études ayant un objectif principal qui n'est pas l'évaluation du risque, notamment l'évaluation de l'efficacité, la description des conditions de prescription et d'utilisation en situation réelle, la pharmacocinétique, l'impact de santé publique peuvent compléter les données de sécurité disponibles. La Table Ronde a analysé les études de sécurité post-AMM des plans de gestion du risque des industriels présents et a dégagé des caractéristiques relatives aux actions proposées.

Quant au cahier des charges et au choix de la méthodologie, seul un canevas général a pu être esquissé compte tenu de la complexité et de la diversité des situations de risque médicamenteux.
\end{abstract}

\section{Introduction}

Depuis 2005 en Europe, les titulaires d'autorisation de mise sur le marché ont l'obligation pour la majorité des nouveaux médicaments de soumettre un plan de gestion de risque dans le même temps que le dossier d'autorisation de mise sur le marché européen, respectant des règles détaillées ${ }^{[1]}$ et un modèle spécifique. ${ }^{[2]}$ Ce dispositif de gestion anticipatrice du risque des médicaments s'appuie sur l'ensemble des données cliniques et non cliniques disponibles et propose des actions de pharmacovigilance, incluant des études complémentaires dites de sécurité et des mesures de minimisation du risque, le cas échéant. ${ }^{[3,4]} \mathrm{Si}$ les études de sécurité après commercialisation représentent un outil important dans

\footnotetext{
* Pour la liste des participants, voir en fin d'article
}

ce système, des interrogations essentielles demeurent. La question se pose en particulier de savoir si les critères de choix méthodologiques pour les études pourraient être résumés dans un cahier des charges en fonction du type de risques et/ou de la nature de l'objectif scientifique (identification ou quantification de certains effets indésirables, évaluation de l'impact des mesures de minimisation des risques ...).

\section{Contexte réglementaire}

Les études de sécurité, interventionnelles ou non, ont comme critère commun de porter sur un médicament commercialisé, utilisé en théorie dans les conditions de l'autorisation de mise sur le marché (AMM). Cette définition et son application pratique 
ont soulevé rapidement des interrogations réglementaires, car la conduite d'une étude observationnelle dans les conditions de l'AMM, n'est pas toujours le reflet de la «vraie vie». Il a fallu attendre la récente révision communautaire pour que cette difficulté soit levée.

\subsection{Révision communautaire}

La directive 2001/83/EC modifiée par la directive 2004/27/CE et le règlement 2004/726/CE ont été révisés dans le cadre du «paquet» pharmaceutique, par la directive 2010/84/EU et le règlement 1235/2010 adoptée le 15 décembre 2010 et publiée au Journal officiel de l'Union européenne le 31 décembre $2010,{ }^{[5,6]}$ ce qui signifie une application du règlement à partir du 12 juillet 2012 et une transposition en droit national de la Directive au plus tard le 21 juillet 2012 .

\subsubsection{Principales dispositions, en dehors des études post-AMM}

Parmi les éléments nouveaux de cette directive, on peut noter concernant la Pharmacovigilance de routine une définition plus large de l'effet indésirable, la possibilité de déclaration par les patients, une détection de signaux renforcée, des changements dans les modalités de réalisation et de soumission des rapports périodiques de Pharmacovigilance (PSUR) et une clarification des procédures pour l'évaluation des données de Pharmacovigilance. Un élément important est la mise en place d'un nouveau comité plus indépendant et plus autonome, et à responsabilités élargies, appelé Pharmacovigilance risk assessment committee (PRAC) qui remplacera le groupe de travail européen de Pharmacovigilance (PhWG, Pharmacovigilance working party), et qui aura pour mandat l'ensemble de la gestion du risque médicamenteux. Par ailleurs des dispositions relatives à la communication et à la transparence ont été intégrées, notamment avec l'établissement d'une liste de médicaments bénéficiant d'une surveillance supplémentaire, la mise en place d'un portail européen avec un lien vers les portails nationaux, la possibilité d'auditions publiques et d'accès aux notifications spontanées contenues dans la base de données de Pharmacovigilance Eudravigilance.

\subsection{2. Études de sécurité post-AMM}

L'article 1 de la Directive introduit une définition plus large des études de sécurité post-AMM : toute étude observationnelle ou interventionnelle portant sur un produit bénéficiant d'une autorisation de mise sur le marché ayant pour but d'identifier, de caractériser ou quantifier un risque, mieux caractériser le profil de sécurité d'emploi d'un médicament, ou de mesurer l'efficacité des mesures de gestion du risque mises en place.

Il est recommandé que les honoraires des professionnels de santé impliqués dans ces études soient limités à la seule compensation du temps passé et des dépenses engagées.

Des dispositions plus précises ont été introduites quant à la validation et au suivi des protocoles des études initiées, pilotées ou financées par le titulaire d'autorisation de mise sur le marché, avec le rôle majeur de coordination et d'évaluation du PRAC (ou de l'autorité nationale s'il s'agit d'une étude menée dans un seul État membre). Des décisions d'actions réglementaires basées sur les résultats de ces études pourront être prises rapidement. Il est également prévu un accès public aux résultats sous forme de résumés disponibles en ligne. Enfin il est important de souligner l'introduction des études d'efficacité post-AMM, qui peuvent être demandées lorsque les données requises ne peuvent être obtenues qu'après l'octroi de l'AMM ou en raison d'avancées scientifiques ou méthodologiques.

\subsection{Futures dispositions nationales}

Sur le plan national, une proposition de loi parlementaire relative aux recherches impliquant la personne humaine, dite « loi Jardé » sera à nouveau débattue devant les parlementaires. ${ }^{\left[{ }^{[7}\right.}$ Elle concerne les recherches organisées et pratiquées sur l'être humain en vue du développement des connaissances biologiques ou médicales. Ce texte est destiné notamment à introduire un cadre réglementaire pour les études non interventionnelles, qui sont caractérisées par le fait que tous les actes sont pratiqués et les produits utilisés de manière habituelle, sans procédure supplémentaire ou inhabituelle de diagnostic, de traitement ou de surveillance. Les principales mesures seraient de disposer d'un avis favorable d'un Comité de protection des personnes (CPP) avant la mise en œuvre de ces études, d'attribuer de nouvelles missions aux CPP se substituant à celles du Comité consultatif sur le traitement de l'information en matière de recherche dans le domaine de la santé (CCTIRS), d'enregistrer ces recherches ainsi que leurs résultats dans un répertoire public, dans un délai raisonnable.

Il sera nécessaire d'être vigilant à la mise en place de ces nouvelles mesures qui devront s'articuler le mieux possible avec les dispositions de la révision communautaire afin de ne pas rendre la France moins attractive que d'autres pays européens, pour conduire des études observationnelles de terrain, en alourdissant notamment les délais de réalisation de ces études, déjà approuvées par le nouveau comité européen PRAC. 


\subsection{Les autres études}

En dehors des études de sécurité après autorisation, il ne faut pas négliger que d'autres études peuvent compléter les données de sécurité disponibles, bien que leur objectif principal ne soit pas l'évaluation du risque mais de l'efficacité, dans le cadre d'une nouvelle indication par exemple, la description des conditions des conditions réelles de prescription ou d'utilisation et de la stratégie thérapeutique, la pharmaco-cinétique ou la pharmacodynamie, la physiopathologie, l'impact de santé publique à travers les différents types d'étude demandées par la Commission de transparence et/ou le Comité d'évaluation des produits de santé, voire aussi des études de marché.

\subsection{Le réseau ENCePP}

Le réseau European Network of Centres for Pharmacoepidemiology \& Pharmacovigilance (ENCePP) est un réseau européen initié par l'agence européenne des médicaments dont l'objectif est de renforcer la surveillance post-AMM des produits de santé après leur mise sur le marché en facilitant la conduite d'études multicentriques, focalisées sur l'évaluation du risque ou du rapport bénéfice/risque. Ce réseau regroupe près de 80 institutions/unités dans le domaine de la pharmacovigilance ou de la pharmaco-épidémiologie qui sont composées de centres de recherche, de centres de soins, de bases de données de santé, de registres électroniques ou de registres portant sur des maladies rares ou des effets indésirables... Depuis 2007, ce réseau s'est attaché à établir une liste de standard méthodologiques ${ }^{[8]}$ et adopter un code de conduite ${ }^{[9]}$ pour promouvoir la transparence et l'indépendance de la recherche conduite au sein du réseau. La liste des standards méthodologiques a été élaborée essentiellement dans la perspective d'améliorer la qualité des études, incitant les protagonistes à respecter certains principes épidémiologiques importants. Le code de conduite couvre des aspects tels que le développement du protocole de l'étude, la conduite de l'étude, la propriété des données et l'accès aux données et à la publication des résultats. En matière de transparence les chercheurs doivent s'engager à publier les résultats d'étude qu'ils soient négatifs ou positifs et rendre publiques des informations pertinentes sur le protocole avant que l'étude ne démarre. Dans le but de faciliter le respect de ces règles, l'European Medicines Agency (EMA) développe actuellement un registre électronique, qui sera accessible au public, destiné à être une référence pour les études de pharmacovigilance ou de pharmaco-épidémiologie menées en Europe. Par ailleurs, en juin 2010 l'Agence européenne des médicaments et le réseau ENCePP ont officiellement lancé le «label ENCePP » qui sera attribué par un comité scientifique aux études de pharmacoépidémiologie répondant aux standards ENCePP. ${ }^{[10]}$

\section{3. État des lieux : analyse des plans de gestion de risque}

\section{1. Méthodes}

Tout d'abord, une revue de la littérature a été réalisée portant sur deux thématiques : l'utilisation, l'impact ou la méthodologie des PASS, et les plans de gestion de risque. Les articles portant spécifiquement sur les bases de données utilisées pour réaliser les PASS ou les articles portant sur des exemples de PASS n'ont pas été retenus.

Parallèlement, une enquête a été réalisée auprès des industriels participant à la Table Ronde afin de recenser et caractériser les PASS prévues au niveau des PGR européens ou demandées par les autorités de santé françaises. En pratique, un tableau a été adressé à chaque laboratoire participant à la table ronde afin de renseigner, pour chaque molécule concernée, les risques recensés au niveau des PGR et leur nature (risque identifié, risque potentiel, information manquante), ainsi que les plans expérimentaux des PASS retenus par les autorités. Pour chaque PASS, les laboratoires devaient préciser s'il s'agissait d'une étude incluant des patients français et les bases de données existantes utilisées le cas échéant.

Enfin, les membres de la Table Ronde ont proposé une synthèse des indications de chaque type d'étude en fonction du type de risque mais aussi de différents critères liés au contexte (fréquence de l'événement, de l'exposition etc.).

\subsection{Revue de la littérature}

Différents types d'actions peuvent être proposées en matière de pharmacovigilance, des notifications spontanées aux PASS. Leur pertinence a été analysée dans le cadre de l'évaluation du rapport bénéfice/risque des médicaments commercialisés. ${ }^{[3,11-13]}$ Les différentes méthodologies utilisées pour les PASS y sont présentées, de la surveillance proactive aux études comparatives, ainsi que des éléments de choix. On peut notamment citer :

- la pharmacovigilance proactive et les données de registres. Dans le cadre des registres, les données sont recueillies de façon systématique sur des patients consécutifs et suivis à long terme;

- les études épidémiologiques descriptives;

- les études épidémiologiques analytiques : elles sont constituées des études cas-témoin, des études de cohorte et des études dérivées (cas propre témoin, ...). Les études de cohorte rétrospectives et les études cas-témoin sont facilitées par un accès à des données existantes recueillies de façon systématique et fiable, par exemple les bases de données 
general ractice research database (GRPD) au Royaume Uni ou PHARMO aux Pays-Bas;

- les essais cliniques (ou extensions d'essais cliniques) spécifiquement conçus pour identifier ou quantifier un ou des risques lié(s) à un produit ayant obtenu l'AMM.

Les PGR détaillent les risques et les engagements en termes d'actions de pharmacovigilance proposés au moment de la demande de mise sur le marché. Frau et al. ont décrit les PGR européens de 15 médicaments approuvés par le Committee for medicinal products for human use (CHMP) dans le cadre d'une procédure centralisée d'AMM entre 2006 et $2007:^{[14]}$ le type de risque (risque identifié, risque potentiel, information manquante) n'était précisé que pour 7 médicaments et des PASS étaient prévues pour 13 médicaments (le nombre total de PASS n'était pas précisé). De même, Giezen et al. ont décrit les PGR européens de 18 médicaments approuvés par le CHMP entre novembre 2005 et mai $2007:{ }^{[11]} 96 \%$ des études avaient un objectif de sécurité et $11 \%$ étaient des études d'utilisation (certaines études ayant un double objectif); $75 \%$ étaient des études de cohorte et $23 \%$ des essais cliniques (notamment des extensions d'essais cliniques); $41 \%$ de ces études étaient des études académiques et $46 \%$ étaient réalisées à partir de données de registres.

Hasford et al. se sont intéressés dès 1998 à l'apport scientifique des PASS. ${ }^{[12]}$ Ils ont étudié 35 études observationnelles réalisées en post-AMM portant sur les domaines de l'hypertension, la psychiatrie, la rhumatologie et l'analgésie, identifiées à partir des publications ou en contactant les industriels. La sécurité constituait un critère de jugement pour 31 de ces études; aucune étude ne comprenait de comparaison à un groupe de référence. Parmi les 21 études renseignant les critères d'éligibilité, 17 présentaient des critères très sélectifs, proches de ceux des essais cliniques, ne permettant donc pas d'appréhender le risque dans les conditions réelles d'utilisation. Le suivi médian était relativement court et non adapté à un risque survenant à distance de l'exposition.

La disponibilité et l'exhaustivité des protocoles des PASS au moment de la présentation du PGR aux autorités de santé dans le cadre de la demande d'AMM ont été analysées par Giezen et al. : ${ }^{[11]} 26 \%$ des PASS présentaient un protocole limité, c'est-à-dire comprenant 11 à 15 des 17 items recommandés par l'International society for pharmacoepidemiology (ISPE). ${ }^{[15]}$ et $74 \%$ des PASS ne proposaient qu'un synopsis (entre 6 et 10 items) ou une description succincte (1 à 5 items), ne permettant pas de juger de la pertinence et de la faisabilité des études proposées. Les auteurs concluent qu'il apparaît nécessaire de faire valider les protocoles par les autorités de santé en prévoyant si possible des échanges entre régulateurs et industriels en amont du processus d'autorisation. Frau et al. ${ }^{[14]}$ ont recherché dans quelle mesure les études prévues étaient finalement menées en recher- chant les publications ou l'état d'avancement de ces études dans les registres d'études clinicaltrials.gov et celui de l'Organisation mondiale de la santé (OMS) en utilisant l'identifiant cité dans le PGR : parmi les 13 médicaments pour lesquels une PASS était proposée, 5 études étaient en cours de réalisation deux ans après la commercialisation. De même, Harmark et al. ont constaté que les études prévues sont rarement mises en place. ${ }^{[3]}$

Le rôle des PGR dans l'amélioration de la gestion du risque est discuté par Frau et al. ${ }^{[14]}$ Parmi les 15 médicaments étudiés, 12 ont présenté des problèmes de sécurité après commercialisation entrainant au total 39 variations de type II (majeures) au niveau du résumé des caractéristiques du produit (RCP) : une seule variation résultait d'un risque confirmé par une PASS prévue au PGR ; 19 variations correspondant à des risques non listés dans le PGR; 13 variations étaient consécutives à un risque confirmé par une PASS non prévue au PGR et 6 variations résultaient de rapports périodiques de sécurité (PSUR) dans le cadre de la pharmacovigilance classique. De plus, les auteurs recensent 9 actions de communications sur la sécurité des produits, qui concernaient 6 de ces 12 médicaments. Ils concluent que les PGR sont peu prédictifs des risques réels observés après commercialisation, que les actions de pharmacovigilance planifiées sont peu suivies en pratique (PASS non réalisées) et que la communication sur les modifications apportées au RCP est insuffisante.

\subsection{Enquête sur les PASS conduites par un échantillon d'industriels dans de cadre des PGR européens}

Parmi les produits commercialisés par 6 laboratoires pharmaceutiques, 33 molécules faisant l'objet d'un PGR ont été recensées dont 8 dans le domaine de l'oncologie, 6 portant sur des biothérapies et 3 sur des vaccins. En moyenne, le nombre de risques notés dans le PGR était de 4,6 (de 1 à 15 selon les molécules). Les types de risques sont distribués de la façon suivante :

- cinquante-sept risques potentiels incluant principalement des événements indésirables spécifiques $(\mathrm{n}=50)$ et des risques de mésusage $(\mathrm{n}=5)$;

- quarante-cinq informations manquantes incluant principalement des populations non incluses $(\mathrm{n}=38)$ et la coadministration avec d'autres molécules $(n=6)$;

- quarante et un risques identifiés incluant principalement des événements indésirables spécifiques.

Cent soixante-dix neuf PASS ont été répertoriées pour identifier, caractériser ou quantifier ces risques. Ces études étaient le plus souvent étiquetées essais cliniques $(n=76$, dont 10 extensions d'essais cliniques), on recense également des études proposant des analyses complémentaires de sécurité réalisées sur les données d'essais en cours $(\mathrm{n}=11)$ ou finalisés $(\mathrm{n}=11)$, des études 
observationnelles comparatives $(\mathrm{n}=31)$ incluant majoritairement des études de cohorte $(\mathrm{n}=24)$, des études observationnelles non comparatives $(n=18)$ avec principalement des études prospectives $(n=16)$, des actions de surveillance active $(n=25$, dont 15 à partir de registres), une méta-analyse et 6 études dont le plan expérimental n'était pas précisé. Seules $29 \%$ de ces études comprenaient des patients français dont une seule était une étude observationnelle comparative.

Cet état des lieux montre une variabilité des types d'études proposés dans le cadre des plans de gestion des risques : les essais cliniques restent largement majoritaires et $76 \%$ des études sont comparatives; on recense très peu d'études cas-témoin ou cas propre témoin et très peu d'études d'utilisation. Les études sont généralement réalisées pour répondre à des objectifs ciblés, on relève très peu d'études visant à l'identification de nouveaux risques.

Les essais cliniques sont davantage proposés dans le cas d'informations manquantes que pour confirmer un risque potentiel ou identifié, ces derniers faisant plutôt l'objet d'analyses complémentaires réalisées à partir d'essais existants. Les études observationnelles comparatives sont à la fois proposées dans le cas d'informations manquantes liées à une exposition non testée (coadministration par exemple) ou pour confirmer un risque. Les actions de surveillance active ainsi que les études d'utilisation (études descriptives) sont principalement proposées pour rechercher un mésusage.

L'interprétation de ces données doit tenir compte du fait qu'elles sont issues d'un échantillon particulier qui n'est pas forcément représentatif de l'ensemble des PGR. De plus, l'analyse de ces PGR a permis de mettre en évidence la confusion qui existe quant à la terminologie utilisée pour définir les types d'études. Ainsi, le terme «étude de cohorte» est indifféremment utilisé pour décrire une étude comparative évaluant un sur-risque en comparant l'incidence d'un événement indésirable chez les patients exposés au médicament à celle de patients non exposés au médicament, ou bien une étude non comparative estimant cette incidence uniquement chez les patients exposés au médicament; dans ce dernier cas, l'absence de groupe de référence ne permet pas de caractériser le sur-risque lié au médicament. De même, l'intitulé « registre » ne permet pas de connaître précisément le type d'étude réalisée à partir des données du registre. Par ailleurs, il est souvent attribué à tort le terme de « registre » à des études de cohorte, qui ne sont pas exhaustive. Enfin, le terme «essai clinique » était utilisé indifféremment pour de réels essais cliniques et pour des extensions d'essais qui correspondent au suivi des patients exposés au médicament et ne comportent donc pas de bras contrôle (il s'agit donc plutôt d'une étude de cohorte sans groupe non exposés, et avec des critères d'éligibilités stricts car ces patients étaient initialement inclus dans un essai clinique).
L'Agence française de sécurité sanitaire des produits de santé (Afssaps) peut demander aux industriels de faire en sorte d'intégrer des centres français au niveau des études demandées par l'EMA dans le cadre des PGR européens. De plus, elle évalue la pertinence de conduire des études uniquement nationales, en fonction des situations spécifiques françaises.

Quand il existe des études supplémentaires, il s'agit principalement d'études d'utilisation; elles permettent de caractériser le profil des prescripteurs et des patients, de connaître les conditions réelles d'utilisation, d'appréhender le risque de pharmacodépendance, d'identifier les abus et l'utilisation hors-AMM. Elles permettent aussi, notamment dans le cadre d'études avant/après, de mesurer l'impact d'une modification du RCP ou de toute autre mesure de minimisation du risque. De façon générale, elles permettent de disposer de données d'utilisation en France.

\section{Indications des différents types d'étude}

En fonction de la spécification des risques mentionnés dans le PGR au moment de la demande d'AMM, certains types d'études sont plus adaptés que d'autres pour évaluer ces risques en postAMM. Pour rappel, les différents types de risque sont les suivants :

- risques identifiés importants : problème de sécurité pouvant avoir un impact sur le rapport bénéfice/risque pour lequel il existe une relation causale avec le médicament est avérée.

- risques potentiels importants : problème de sécurité pouvant avoir un impact sur le rapport bénéfice/risque pour lequel l'association avec le médicament est évoquée, mais doit être confirmée.

- informations manquantes importantes : l'information n'est pas disponible au moment de la demande d'AMM (population particulière, co-administration possible avec un autre produit,...).

Le tableau I présente les indications des différents types d'études telles que définies lors de la Table Ronde.

\section{Conclusion}

Dans notre étude de cas, il apparaît que les PASS menées à ce jour dans le cadre de PGR européens sont majoritairement des essais cliniques (ou extensions d'essais cliniques), dans une moindre mesure des études observationnelles, et que moins d'un tiers de ces études incluent des patients français. L'état des lieux montre une hétérogénéité dans de la terminologie utilisée pour dénommer les types d'études. Il apparaît important qu'un effort de standardisation dans l'utilisation de ces termes soit réalisé. En dehors 
Tableau I. Indications, avantages et limitations des types d'études en pharmaco-épidémiologie

\begin{tabular}{|c|c|c|c|}
\hline Nature des études & Indication & Avantages & Limites \\
\hline Notification spontanée & $\begin{array}{l}\text { - Risque non connu et faible } \\
\text { bruit de fond } \\
\text { - Génération de signal }\end{array}$ & $\begin{array}{l}\text { - Système en place } \\
\text { - Produits remboursés ou non } \\
\text { - Réglementaire }\end{array}$ & $\begin{array}{l}\text { - Sous déclaration } \\
\text { - Dénominateur incertain } \\
\text { - Inefficace si bruit de fond élevé } \\
\text { - Inadapté si délai de survenue } \\
\text { important et risque non identifié }\end{array}$ \\
\hline
\end{tabular}

\begin{tabular}{lll}
\hline Notification organisée & $\bullet$ Évaluation de l'incidence & $\bullet$ Minimisation de la sous \\
Ex : Prescription Event & d'un EI & notification \\
Monitoring & - Fréquence de l'EI modérée & $\bullet$ Permet de calculer des taux \\
& ou élevée & d'incidence \\
& - Produit bien implanté sur & $\bullet$ Rapide \\
& le marché & $\bullet$ Peu coûteux
\end{tabular}

- Nécessite une pénétration

suffisante du produit sur le marché

- Recueil d'information sur un échantillon

- Biais de sélection des médecins et des patients

- Biais de mesure

\begin{tabular}{|c|c|c|c|}
\hline Étude transversale & $\begin{array}{l}\text { - } \text { Caractérisation de la } \\
\text { population rejointe } \\
\text { - Identification de l'usage } \\
\text { hors AMM }\end{array}$ & $\begin{array}{l}\text { - Facile à mettre en œuvre, } \\
\text { rapide } \\
\text { - Relativement peu coûteuse }\end{array}$ & $\begin{array}{l}\text { - Ne permet pas la quantification du } \\
\text { risque } \\
\text { - N'intègre pas la temporalité du } \\
\text { risque }\end{array}$ \\
\hline $\begin{array}{l}\text { Cohorte non comparative } \\
\text { prospective }\end{array}$ & $\begin{array}{l}\text { - Estimation de l'incidence } \\
\text { de l'EI en situation réelle } \\
\text { - Identification de } \\
\text { sous-populations à risque }\end{array}$ & $\begin{array}{l}\text { - Relativement rapide à mettre } \\
\text { en place dès la mise sur le } \\
\text { marché } \\
\text { - Représentatif de la population } \\
\text { traitée en vie réelle } \\
\text { - Recueil exhaustif de } \\
\text { l'information possible }\end{array}$ & $\begin{array}{l}\text { - Biais de sélection des médecins et } \\
\text { des patients } \\
\text { - Comparaisons : par approches } \\
\text { indirectes très biaisées } \\
\text { - Coût notable } \\
\text { - Peu adapté pour des risques rares } \\
\text { ou à latence longue } \\
\text { - Perdus de vue }\end{array}$ \\
\hline $\begin{array}{l}\text { Cas / témoins spécifique } \\
\text { Cas / propre témoin }\end{array}$ & $\begin{array}{l}\text { - Évaluation d'un sur-risque } \\
\text { - Toutes fréquences d'EI } \\
\text { y compris risque rare } \\
\text { - Exposition suffisamment } \\
\text { fréquente }\end{array}$ & $\begin{array}{l}\text { - Permet d'étudier plusieurs } \\
\text { facteurs de risque } \\
\text { - Rapide et moins coûteux } \\
\text { qu'une étude de cohorte }\end{array}$ & $\begin{array}{l}\text { - Choix des témoins : doivent avoir } \\
\text { la même probabilité d'être expo- } \\
\text { sés au médicament que les cas } \\
\text { - Mesure d'exposition difficile } \\
\text { (biais de mémorisation, difficile si } \\
\text { latence longue de l'événement) } \\
\text { - Ne permet l'étude que d'un EI }\end{array}$ \\
\hline $\begin{array}{l}\text { Cohorte (exposé / non } \\
\text { exposé) prospective } \\
\text { spécifique }\end{array}$ & $\begin{array}{l}\text { - Évaluation d'un sur-risque } \\
\text { - Fréquence modérée à } \\
\text { élevée } \\
\text { de l'EI, quel que soit le bruit } \\
\text { de fond } \\
\text { - Exposition rare possible }\end{array}$ & $\begin{array}{l}\text { - Recueil exhaustif des } \\
\text { événements } \\
\text { - Représentatif de la population } \\
\text { dans la vie réelle } \\
\text { - Mesure non biaisée de } \\
\text { l'exposition } \\
\text { - Permet la quantification } \\
\text { comparative du risque } \\
\text { - Étude de plusieurs risques }\end{array}$ & $\begin{array}{l}\text { - Choix du/des comparateur(s) } \\
\text { difficile } \\
\text { - Biais de déplétion des susceptibles } \\
\text { (si patients prévalents), facteurs de } \\
\text { confusion (ajustement), biais d'in- } \\
\text { dication } \\
\text { - Peu efficace pour des risques rares } \\
\text { - Long et coûteux } \\
\text { - Perdus de vue } \\
\text { - Ne permet l'étude que d'une expo- } \\
\text { sition }\end{array}$ \\
\hline $\begin{array}{l}\text { Étude sur base de données } \\
\text { médicales ou de rembour- } \\
\text { sement (cohorte ou étude } \\
\text { cas témoin) }\end{array}$ & $\begin{array}{l}\text { - Idem cohortes compara- } \\
\text { tives et études cas témoins } \\
\text { - Quantification d'un sur- } \\
\text { risque même rare (pour } \\
\text { un produit prescrit pour } \\
\text { les BD médicales et rem- } \\
\text { boursé pour les BD de } \\
\text { remboursement) } \\
\text { - Produit bien établi sur le } \\
\text { marché }\end{array}$ & $\begin{array}{l}\text { - Rapide à réaliser } \\
\text { - Peu coûteux } \\
\text { - Moins de biais de sélection et } \\
\text { pas de biais de mesure } \\
\text { - Comparaison avec d'autres } \\
\text { classes thérapeutiques possibles } \\
\text { - Puissance importante }\end{array}$ & $\begin{array}{l}\text { - Données limitées à ce qui est dis- } \\
\text { ponible dans la base, donc défauts } \\
\text { d'ajustement } \\
\text { - Biais difficiles à estimer } \\
\text { - Problème d'accessibilité } \\
\text { - Problème de qualité / données } \\
\text { - Manquantes } \\
\text { - Multiplicité des tests d'hypothèse }\end{array}$ \\
\hline
\end{tabular}


Tableau II. Suite.

\begin{tabular}{|c|c|c|c|}
\hline Nature des études & Indication & Avantages & Limites \\
\hline $\begin{array}{l}\text { Essai randomisé de } \\
\text { sécurité }\end{array}$ & $\begin{array}{l}\text { - Quantification d'un excès } \\
\text { de risque par rapport à un } \\
\text { traitement de référence } \\
\text { - Fréquence élevée ou modé- } \\
\text { rée de l'EI }\end{array}$ & $\begin{array}{l}\text { - Comparabilité des groupes (ran- } \\
\text { domisation); limite au maximum } \\
\text { les biais (surtout si double insu). } \\
\text { - Efficace si bruit de fond élevé }\end{array}$ & $\begin{array}{l}\text { - Représentativité de la population } \\
\text { traitée } \\
\text { - Long, coûteux } \\
\text { - Problème d'acceptabilité par les } \\
\text { comités d'éthique et les investiga- } \\
\text { teurs si risque déjà identifié }\end{array}$ \\
\hline Méta-analyse de sécurité & $\begin{array}{l}\text { - Quantification d'un excès } \\
\text { de risque par rapport à un } \\
\text { traitement de référence } \\
\text { - Fréquence modérée à éle- } \\
\text { vée, quel que soit le bruit de } \\
\text { fond }\end{array}$ & $\begin{array}{l}\text { - Puissant (nombre de patients } \\
\text { élevé), } \\
\text { - Limite les biais, } \\
\text { - Peu coûteux (plus si données in- } \\
\text { dividuelles). } \\
\text { - Identification de sous-groupes à } \\
\text { risque si données individuelles. } \\
\text { - Efficace si bruit de fond élevé }\end{array}$ & $\begin{array}{l}\text { - Agrégation de données d'essais } \\
\text { randomisés, (variabilité : critères } \\
\text { d'éligibilité, doses, durées, re- } \\
\text { cueil). } \\
\text { - Qualité moindre des données de } \\
\text { sécurité, données individuelles né- } \\
\text { cessaires pour regrouper les codes } \\
\text { diagnostiques } \\
\text { - Résultats quelquefois discordants } \\
\text { selon études incluses } \\
\text { - Représentatif de la population des } \\
\text { essais randomisés }\end{array}$ \\
\hline
\end{tabular}

AMM : autorisation de mise sur le marché ; BD : base de données ; EI : événement indésirable ; PV : pharmacovigilance ; RCP : résumé des caractéristiques des produits

des PASS prévues dans le cadre des PGR européens, des études post-AMM peuvent être demandées par les autorités de santé nationales et sont principalement des études d'utilisation et d'impact en santé publique, dont certaines comportent des données de sécurité. Par ailleurs, même si cela pourrait s'avérer difficile dans certaines situations (place incertaine du médicament dans l'arsenal thérapeutique ...), les protocoles des PASS devraient pouvoir être validés par les autorités de santé au moment de la demande d'autorisation de mise sur le marché.

Pour guider le choix de la méthode de ces études, les participants à la Table Ronde rappellent qu'il n'y a pas de solution univoque, et qu'il faut tenir compte des paramètres suivants : la population cible, l'exposition (fréquence, condition d'accès au produit, indications et contre indications, positionnement dans la stratégie thérapeutique), le risque (fréquence et nature, risque de base dans la population concernée, délai de latence, identification du risque potentiel au moment de la commercialisation ou ultérieurement). La décision doit s'appuyer sur le niveau de preuve des études possibles mais également sur les paramètres de faisabilité.

Si un cahier des charges détaillé pour la réalisation pratique et les choix méthodologiques n'a pu être élaboré pour ces études au regard de la complexité et de la diversité des situations de risque médicamenteux, l'esquisse d'un tel cahier a cependant pu être dressé sous la forme d'un canevas général. Plusieurs études et plusieurs schémas d'études sont parfois nécessaires pour répondre à une question de sécurité. Chaque situation est particulière, il est donc nécessaire de recourir à une expertise pharmacoépidémiologique.

\section{Participants}

Nous remercions chaleureusement les membres de la Table Ronde qui ont participé à cette synthèse.

Laurent Auclert (Sanofi-Aventis), Marthe Bonnin (GSK), Magda Daudin (Afssaps, Saint Denis), Catherine Dubois (Roche), Alain Francillon (Novartis), Elisabeth Frauger, Danièle Girault (Novartis), Marie-Laurence Gourlay (Afssaps, Saint Denis), Pascale Jolliet (CRPV, Nantes), Carmen Kreft Jaïs (Afssaps, Saint Denis), Lamiae Grimaldi (LA-SER), Michel Lièvre (CHU, Lyon), Patricia Maillère (Servier), Philippe Maugendre (Sanofi-Aventis), Joelle Micallef (CHU, Marseille), Sara Miranda (Afssaps, Saint Denis), Antoine Pariente (CHU Bordeaux), Sylvie Paulmier Bigot (Leem), Jonathan Pentel (Lilly), Laure Prestat (Cnamts, Paris), Fanny Pruvot (BMS), Valérie Querol Ferrer (Astra Zeneca), Fanny Rocher (CRPV, Nice), Christel Saussier (Afssaps, Saint Denis), Laura Zanetti (HAS, Saint Denis La Plaine). 


\section{Références}

1. Committee for Medicinal Products for Human Use. Guideline on risk management systems for medicinal products for human use. 2009 http://ec. europa.eu/health/documents/eudralex/vol-9/ index_en.htm

2. Committee for Medicinal Products for Human Use. Annex C: template for EU risk management plan (EMEA/192632/2006). 2006 http:// eudravigilance. ema . europa. eu/human/docs/19263206en.pdf

3. Harmark L, van Grootheest AC. Pharmacovigilance: methods, recent developments and future perspectives. Eur J Clin Pharmacol 2008; 64: 743-52

4. Hartford CG, Petchel KS, Mickail H, et al. Pharmacovigilance during the preapproval phases: an evolving pharmaceutical industry model in response to ICH E2E, CIOMS VI, FDA and EMEA/CHMP risk-management guidelines. Drug Saf 2006; 29: 657-73

5. Rectificatif à la directive 2010/84/UE du Parlement européen et du Conseil du 15.12.2010 modifiant en ce qui concerne la Pharmacovigilance la directive 2001/83/CE instituant un code communautaire relatif aux medicaments à usage humain, JOUE L 348 du 31 décembre 2010. http: //eur-lex . europa.eu/fr/index.htm

6. Règlement UE $\mathrm{n}^{\circ} 1235 / 2010$ du Parlement européen et du Conseil du 15.12.2010 modifiant la pharmacovigilance des médicaments à usage humain, le règlement $(\mathrm{CE}) \mathrm{n}^{\circ} 726 / 2004$ établissant des procédures communautaires pour l'autorisation et la surveillance en ce qui concerne les médicaments à usage humain et à usage vétérinaire et instituant une Agence européenne des médicaments et le règlement (CE) n 1394/2007 concernant les médicaments de thérapie innovante, JOUE L 348 du 31 décembre 2010. http: //eur-lex. europa.eu/fr/index.htm

7. Proposition de loi relatives aux recherches impliquant la personne humaine, $n^{\circ} 426$, Sénat. http: //www. senat. fr
8. The check list of methodological standards for ENCePP study protocols. http://www . encepp.eu/standards_and_guidances/index.html

9. The ENCePP code of conduct. 2010 http://www . encepp. eu/documents/ encepp_studies/ENCePP\%20Code\%20of\%20Conduct_20100507.pdf

10. Press release European Medicines Agency and European network of Centres for Pharmacoepidemiology \& Pharmacovigilance (ENCePP) launch ENCePP studies (EMA/343605/2010). http://www. ema. europa. eu/docs/en_GB/document_library/Press_release/2010/06/ WC500091825.pdf

11. Giezen TJ, Mantel-Teeuwisse AK, et al. Evaluation of post-authorization safety studies in the first cohort of EU Risk Management Plans at time of regulatory approval. Drug Saf 2009; 32: 1175-87

12. Hasford J, Lamprecht T. Company observational post-marketing studies: drug risk assessment and drug research in special populations-a study-based analysis. Eur J Clin Pharmacol 1998; 53: 369-71

13. Naldi L. How to evaluate the benefit-risk profile of newly marketed drugs. Ann Dermatol Venereol 2010; 137: 264-6, 1-3

14. Frau S, Font Pous M, Luppino MR, et al. Risk Management Plans: are they a tool for improving drug safety? Eur J Clin Pharmacol 2010; 66: 785-90

15. ISPE. Guidelines for good pharmacoepidemiology practices (GPP). Pharmacoepidemiol Drug Saf 2008; 17: 200-8

Correspondance et offprints : Véronique Lamarque-Garnier, Direction Évaluation de Santé, Sécurité et Gestion du Risque, Laboratoire Pfizer, 23-25 avenue du Docteur Lannelongue, 75768 Paris cedex 14, France. E-mail : veronique.lamarque@pfizer.com 